Instituto Internacional de Investigación y Desarrollo Tecnológico Educativo INDTEC, C.A.

DOI: https://doi.org/10.29394/Scientific.issn.2542-2987.2020.5.17.19.350-369

OAI-PMH: http://www.indteca.com/ojs/index.php/Revista Scientific/oai

Ensayo Original / Original Essay

\title{
Aprendizaje mediante el uso de Herramientas Tecnológicas en la Educación inclusiva y el fortalecimiento de la enseñanza
}

\author{
Autoras: Silvia Yesenia Andrade Parra \\ Universidad Nacional de Educación, UNAE \\ silvi yesse01@outlook.es \\ Azogues, Ecuador \\ https://orcid.org/0000-0001-8549-377X \\ Martha Jhamiled Tapia Tapia \\ Universidad Nacional de Educación, UNAE \\ ihamytapia@hotmail.com \\ Azogues, Ecuador \\ https://orcid.org/0000-0002-8439-9172 \\ Flor del Cisne Tituana Vásquez \\ Universidad Nacional de Educación, UNAE \\ tituanaflor@gmail.com \\ Azogues, Ecuador \\ https://orcid.org/0000-0003-1586-690X
}

\section{Resumen}

El sistema educativo ecuatoriano actual se encuentra frente a una variedad de retos, motivo por el cual se desarrolla esta investigación para analizar la importancia de la implementación de los procesos tecnológicos en la mejora de la enseñanza-aprendizaje ante esta emergencia que estamos atravesando donde nos encontramos en un confinamiento, donde resulta necesario replantear las estrategias y métodos más adecuados que atiendan a la diversidad de cada estudiante. Así como valorar el rol fundamental que desempeña el docente como mediador de este proceso y la necesidad de acceder a diversas capacitaciones que le permitan hacer uso correcto de las tecnologías y por ende atender los intereses de los estudiantes. Es un reto que se debe enfrentar, en efecto el siguiente trabajo presenta los avances que han surgido en cuanto a las TIC-TAC y TEP como tendencias educativas, que cambian las concepciones tradicionales, convirtiéndose en herramientas para aprender a aprender; encaminados a una educación de calidad y éxito en cada estudiante.

Palabras clave: enseñanza; aprendizaje; docente; estudiante; tecnología.

Cómo citar este ensayo:

Andrade, S., Tapia, M., \& Tituana, F. (2020). Aprendizaje mediante el uso de Herramientas Tecnológicas en la Educación inclusiva y el fortalecimiento de la enseñanza. Revista Scientific, 5(17), 350-369, e-ISSN: 2542-2987. Recuperado de: https://doi.org/10.29394/Scientific.issn.25422987.2020.5.17.19.350-369

Fecha de Recepción: 25-03-2020
Fecha de Aceptación: 22-06-2020
Fecha de Publicación: 05-08-2020 


\title{
Learning through the use of Technological Tools in inclusive Education and the strengthening of teaching
}

\begin{abstract}
The current Ecuadorian educational system is facing a variety of challenges, which is why this research is being carried out to analyze the importance of the implementation of technological processes in improving teaching-learning in the face of this emergency that we are going through where we are. in a confinement, where it is necessary to rethink the most appropriate strategies and methods that attend to the diversity of each student. As well as assessing the fundamental role that the teacher plays as a mediator of this process and the need to access various trainings that allow them to make correct use of technologies and therefore serve the interests of students. It is a challenge that must be faced, in effect the following work presents the advances that have emerged in terms of ICT-TAC and PET as educational trends, which change traditional concepts, becoming tools to learn to learn; aimed at a quality education and success in every student.
\end{abstract}

Keywords: teaching; learning; teacher; student; technology.

\footnotetext{
How to cite this essay:

Andrade, S., Tapia, M., \& Tituana, F. (2020). Learning through the use of Technological Tools in inclusive Education and the strengthening of teaching. Revista Scientific, 5(17), 350-369, e-ISSN: 2542-2987. Recovered from: https://doi.org/10.29394/Scientific.issn.2542-2987.2020.5.17.19.350$\underline{369}$
}

Date Received: 25-03-2020
Date Acceptance: 22-06-2020
Date Publication: 05-08-2020 


\section{Introducción}

Ante la realidad que atraviesa el mundo las tecnologías forman parte de las actividades que desarrollamos a diario, es por lo que yace la necesidad de incorporarlas en las diferentes estrategias y métodos para complementar la enseñanza-aprendizaje de nuestros estudiantes hoy en día y que estas respondan a los diferentes interesen que poseen.

En la educación el docente cumple un rol fundamental, motivo por el cual debe poseer los conocimientos acordes de los adelantos que surgen día a día en la tecnología y al no ser así debe existir el compromiso para acceder a cursos de capacitación que le permitan conocer y utilizar las diferentes herramientas tecnológicas. Ya que el docente en esta época es guía para el desarrollo de los aprendizajes ante un público que pertenece a una era digital.

Al tener a nuestra disposición un sinnúmero de herramientas tecnológicas debemos hacer uso de estas para mejorar nuestra labor docente y generar aprendizajes significativos en los estudiantes, que les permitan interactuar de una manera oportuna y sean más autónomos y creativos en el desarrollo de sus aprendizajes.

Por lo tanto, conocer e implementar el uso de estas herramientas tecnológicas, para trabajar con la diversidad e incluir, es posible, gracias a algunas de las herramientas que en este artículo se exponen y entre ellas tenemos: Las TIC, TAC y TEP como recursos en el aprendizaje.

\section{Desarrollo}

\subsection{La educación}

La educación ha pasado por una serie de cambios desde muchos años atrás, donde se podía apreciar la segregación de varios estudiantes, poco a poco se fue realizando cambios y se llegó a una integración dentro de las aulas educativas, pero la integración no es suficiente la realidad actual demanda de una verdadera educación inclusiva que permita al estudiante formar parte de 
la sociedad en la que se desenvuelve.

Es por ello que necesitamos una sociedad cambiante hacia una mejora que permita garantizar el derecho a la educación que tiene cada individuo, la Educación Inclusiva supone conseguir que todos los individuos tengan acceso a igualdad y equidad de oportunidades en los diferentes ámbitos y contextos, brindándoles a los estudiantes las herramientas necesarias para afrontarse a las diversas situaciones del cotidiano vivir.

Se debe considerar que en el proceso educativo a los estudiantes de hoy en día se los denominen nativos digitales al respecto Prensky (2001), citado por García, Gértrudix, Durán, Gamonal y Gálvez (2011): quien "define a los nativos digitales como la primera generación que ha crecido con las tecnologías digitales y que son "nativos" del lenguaje de los ordenadores, videojuegos e Internet [...]" (pág. 112). Necesitamos transformar el Sistema Educativo, que permita a los niños conseguir el trabajo que buscan y no encuentran, debilitando sus sueños y aspiraciones en el futuro; por lo tanto, se debe reflexionar en cómo conseguir que los estudiantes estén motivados, interesados en obtener su propio aprendizaje.

\subsection{TIC}

Hoy en día las tecnologías nos proporcionan gran variedad de recursos que permitirán generar cambios y proporcionar mejores aprendizajes a nuestros estudiantes, atendiendo cada una de sus necesidades, a través de información audiovisual, multimedia y más, así también nos brindan la facilidad de una comunicación más rápida con los diferentes actores educativos.

Al respecto, Belloch (2012a), afirma que las TIC se definen como: "Tecnologías para el almacenamiento, recuperación, proceso y comunicación de la información" (pág. 2); y nos ofrecen varias herramientas que nos permiten desarrollar diversas actividades de una manera más ágil y oportuna.

Dentro de este grupo existen varios dispositivos que nos generan esta 
información entre ellos tenemos el televisor, el teléfono, la tablet, la computadora. Actualmente los dispositivos que más se ocupan son las laptops y los celulares. Internet nos proporcionan variedad de información, programas multimedia, diversas aplicaciones y las diferentes redes sociales que nos permiten interactuar y estar en constante comunicación.

De acuerdo con Martínez (2018): se debe considerar a las TIC en "la elaboración de contenidos divulgación de trabajos y tareas, la resolución de problemas y la toma de decisiones, en medio de un clima colaborativo, cooperativo, enriquecedor y motivante que invita a seguir adelante en forma individual y grupal" (págs. 158-159). Si se busca atender a la diversidad e incluir a todos los estudiantes se debe fomentar, gracias a las TIC el trabajo cooperativo, en pares, que se apoyen los unos a los otros mediante las nuevas herramientas para el aprendizaje.

Es así como la Web ha evolucionado de una manera vertiginosa en varios aspectos como lo son la gran cantidad de usuarios que en la actualidad acceden a los diferentes servicios que ofrece, la interacción, la rapidez en el acceso, los diferentes contextos entre otros.

Se dio inicio con la Web 1.0 dónde se realizó un consumo pasivo en medios como la radio y televisión. Con la llegada de la Web 2.0 se ha creado una revolución en la educación, pues es una gran fuente de conocimiento, nos permite usar una infinidad de recursos y con estos potenciar el autoaprendizaje, el trabajo cooperativo y colaborativo, hacer que los alumnos se conviertan en usuarios activos y dejen de ser consumidores pasivos que solo receptan los conocimientos impartidos por los docentes. Además, con el uso del internet el alumnado aprende y refuerza los conocimientos que se trabajan en clase.

El uso de la Web 2.0 en el aula, propicia un ambiente dinámico, didáctico, ayuda y motiva no solo al alumnado sino también a los docentes, nos permite resolver dudas, ampliar información sobre contenidos que se 
estén trabajando. Asimismo, brinda al estudiante el papel protagónico de su aprendizaje.

Otro aspecto importante de la Web 2.0 es que es un medio que permite alcanzar destrezas digitales, los estudiantes crean blogs, videos, juegos, las wikis etc., interactúan con individuos que se encuentran en diferentes contextos a nivel mundial, esta competencia digital es necesaria para desempeñarse efectivamente ante las exigencias de la sociedad tecnológica, en la que esperamos se desenvuelvan profesionalmente en el futuro.

La era 2.0 ha influido en el desarrollo de la educación, por ende, los profesores necesitan estar capacitados en el uso de las nuevas tecnologías digitales y del internet, este conocimiento y preparación permanente, les permitirá enseñar de una mejor manera. Un gran desafío es aprovechar esas herramientas tecnológicas de manera innovadora y creativa.

Los docentes deben incorporar estas herramientas para lograr que el alumnado desarrolle una serie de capacidades cognitivas, fomente su autonomía, su capacidad para investigar y procesar la información, compartir contenidos intelectuales, etc.

Por lo tanto, es importante la preparación de los maestros en la implementación de las TIC y la transformación del modelo enseñanzaaprendizaje tanto para el profesor como para el alumno. Pues en esta era digital en la que vivimos, nos ha tocado enfrentar nuevos retos debido a la pandemia del COVID-19 se ha podido evidenciar la poca o nula preparación del profesorado en el manejo y uso de la tecnología. Resulta fundamental capacitarnos en base a la utilización de estas herramientas tecnológicas y así poder atender los requerimientos, necesidades e intereses de nuestros estudiantes en la actualidad.

Paralelamente Belloch (2012b): también nos habla de la "Web 3.0. Las innovaciones que se están produciendo en estos momentos se basan en Sociedades Virtuales, realidad virtual, web semántica, búsqueda inteligente" 
Instituto Internacional de Investigación y Desarrollo Tecnológico Educativo INDTEC, C.A.

DOI: https://doi.org/10.29394/Scientific.issn.2542-2987.2020.5.17.19.350-369

OAI-PMH: http://www.indteca.com/ojs/index.php/Revista_Scientific/oai

Ensayo Original / Original Essay

(pág. 6); y es la realidad que estamos atravesando al encontrarnos aislados en nuestros hogares, estamos desarrollando casi en la totalidad nuestras actividades de manera virtual, nos hemos convertido en sociedades virtuales.

\subsection{TAC}

Las TAC son aquellas herramientas que van a generar esos conocimientos y van a permitir a los usuarios adquirir los mismos, es decir van a dar respuesta a lo que demanda la sociedad actual y formar parte de esta sociedad del conocimiento. Frente a esto, Martínez (2016a), indica que:

Las Tecnologías de la Aprendizaje y la Comunicación (TAC) están transformando de manera vertiginosa nuestros modos de vida. Han impuesto la alfabetización científico - tecnológica de todos los ciudadanos y se ha dado un uso intensivo de las tecnologías en muchas actividades habituales. Por esta razón, están produciendo una auténtica revolución en la vida del ser humano, que se enmarca en un conjunto más amplio de cambios en nuestra sociedad y se concreta en una denominación: la sociedad de la información (pág. 94).

La educación virtual ha dado un gran cambio, gracias a la aparición de la era digital que dio variedad de recursos para cambiar la educación tradicional. Entre estos recursos, el Celular, la Tablet y Laptop se convierten en dispositivos multifuncionales, ya que permiten acceder a ver la televisión, navegar por Internet, jugar video juegos, etc.

Según Arza (2010): "los videojuegos son programas informáticos de entretenimiento que pueden ser utilizados a través de diferentes canales" (pág. 29); como computadores y consolas. En el computador pueden ser instalados a través de un $C D$ o descargados del internet, aunque existe también diversidad de video juegos en línea, que permiten la interacción simultánea con otras personas que estén conectadas en ese mismo momento.

Al existir una gran variedad de juegos no todos ejercen una influencia negativa, hay algunos que permiten el aprendizaje de materias, valores, etc. 
Que son los que se debería considerar dentro del ámbito educativo para poder responder a los intereses de la sociedad actual.

Por esta razón, los videojuegos son una forma de diversión y alegría, proporcionando estructura y disciplina la misma que tiene objetivos claros que impulsan a la motivación. Se ha evidenciado que ciertos profesionales destacados de medicina entre edades de 30 y 40 años juegan videojuegos y al realizar una cirugía laparoscópica se desempeñan muy bien en su labor.

Al respecto en una investigación realizada por Roncancio-Ortiz, OrtizCarrera, Llano-Ruiz, Malpica-López y Bocanegra-García (2017): evidencian "[...] que estas herramientas tecnológicas pueden ser utilizadas como mecanismos didácticos que ayudan, entre otras cosas, a la resolución de problemas de aprendizaje, al mejoramiento de las habilidades motoras y cognitivas, y al fomento de la creatividad" (pág. 36). Entonces es valioso entender que se debe utilizar las herramientas tecnológicas que son de libre acceso para fortalecer las habilidades antes mencionadas; ya que los niños de hoy en día o llamados nativos digitales, han nacido y se desenvuelven en esta realidad, donde los docentes debemos realizar los ajustes pertinentes para aprovechar de mejor manera estas habilidades.

Otro recurso que nos ofrece el internet, son los blogs que señalando a Matheson (2004), citado por Martín y Montilla (2016), manifiesta que: "los blogs son publicaciones online que el usuario puede actualizar constantemente" (pág. 662); poseen entradas cronológicas, con una interfaz sencilla que facilita su uso de forma interactiva para que los usuarios puedan responder de manera cómoda e inmediata.

La Wiki es otro recurso de gran importancia. Conforme a Mora (2012), la Wiki: "posee características que lo hacen un medio eficaz para desarrollar trabajos colaborativos y propiciar un papel activo por parte del usuario, ya que es posible modificarlo las veces que sea necesario e interactuar con distintos participantes de forma asincrónica" (pág. 113). Los Videojuegos, los blogs, las 
Wikis son herramientas que proporcionan información y permiten interactuar a una gran cantidad de usuarios, a la vez que comparten sus diferentes experiencias y aportaciones por medio de estos espacios, lo que genera, aprendizajes de manera innovadora, autónoma y creativa.

\subsection{TEP}

Explican Zambrano y Balladares (2017a): que "[...] es una nueva terminología que se le asigna a las tecnologías que se utilizan como sustento para la cohesión social de un grupo determinado de personas [...]" (pág. 169). Por consiguiente, nos permite mejorar las condiciones de vida, permitiendo un aprendizaje autónomo activo, desarrollando las habilidades individuales en la conformación de un contexto participativo; fomentando la creatividad colectiva.

El Ministerio de Tecnologías de la Información y Comunicaciones de Colombia (2015), citado por Becerra (2019): se refiere a la clasificación de las TEP en "[...] el uso de las redes sociales como espacios de participación ciudadana, mostrando una posición activa en la cual impulsan cambios positivos frente a asuntos de interés comunitario o causas solidarias" (pág. 59). Siendo de gran utilidad en la época que nos encontramos inmersos, de cambios constantes y como educadores tenemos que capacitarnos, investigar y poner en marcha todas las herramientas que están a nuestro alcance.

Por lo antes dicho el rol del docente debería ser el de empoderarse y convertirse en un experto en cuanto a la participación, pues ser consciente de que esta encargado de las habilidades de cada estudiante, de conocer la diversidad de su aula y las inteligencias múltiples que estos poseen, de que cada uno de sus estudiantes se contagien de este empoderamiento individual, es decir, que ellos tomen las riendas de su aprendizaje en cuanto a ser innovadores, creativos y reflexivos mediante el uso que den a las tecnologías.

En este sentido, Zambrano y Balladares (2017b): indican que "hablamos de una red social en la cual se fomenta la participación activa de 
los usuarios en temas relacionados con política, sociedad y/o educación [...]" (pág. 169). Con respecto al uso de las redes sociales, son de suma importancia hoy en día debido a que por estos medios se están manejando las clases ya sea para impartirlas o la recepción de las tareas; también se puede constatar que el uso de estas herramientas motiva a los estudiantes a realizar sus tareas, recordando que se trabaja con nativos digitales, pues se sienten a gusto y cómodos al manejar estos recursos.

El docente tiene que actualizarse en el uso de estas herramientas para poder brindar un mejor asesoramiento y servicio a los estudiantes y así poder satisfacer las dudas que tengan, es necesario tener en cuenta desde que contexto se está trabajando ya que lamentablemente en la zona rural hay mayores inconvenientes que en la urbana, urgiendo la preparación de todos los maestros en cuanto al empoderamiento y uso de estas herramientas para mejorar la calidad educativa.

En conformidad con Cabero y Fernández (2014): “[...] encontramos con tres diferentes formas de aplicarlos, no contrapuestas sino complementarias: TIC (Tecnologías de la Información y la Comunicación), TAC (Tecnologías para el Aprendizaje y el Conocimiento) y TEP (Tecnologías para el Empoderamiento y la Participación)" (pág. 38). Los diferentes tipos de tecnologías nos facilitan las herramientas que nos van a permitir una mejor interacción social, a la vez que se construye el conocimiento, y se atiende las diferencias individuales de cada estudiante.

En la actualidad muchos docentes aún no han adoptado la tecnología como se debería, especialmente en estos momentos de emergencia sanitaria que estamos viviendo, hoy más que nunca necesitamos conocer estas herramientas que nos van a permitir desarrollar las clases de una manera creativa e innovadora y así poder llegar a nuestros estudiantes. En este mundo cambiante las tecnologías han invadido cada uno de nuestros hogares y nosotros debemos ir al nivel de estos. 
También tenemos la educación a distancia, que brinda grandes ventajas a las personas para que puedan acceder a la educación y estudiar la carrera que desean desde la comodidad de sus hogares y a la medida de sus ritmos y sus tiempos. De igual manera, permite la cooperación con los demás, al acceder a información de apoyo, incluso para aprender otros idiomas y leer; debemos de reflexionar que la tecnología ahora es parte de nuestro mundo y del mundo del trabajo, es imprescindible que la educación se adapte a las necesidades de los estudiantes que día a día exigen más el uso de estas herramientas, pero por otro lado, los estudiantes también tienen que ser responsables del uso que den en su aprendizaje y de los peligros a los que se exponen al no utilizar adecuadamente estas herramientas.

Adicionalmente Forero (2009), aclara que:

El impacto de las nuevas tecnologías se ha extendido paulatinamente al conjunto de la sociedad, la política, la educación, la comunicación, el entretenimiento, y, en general, a la manera como las personas ven el mundo y se perciben a sí mismas (pág. 41).

\subsection{La enseñanza-aprendizaje}

Como presenta Brito (2004):

En el marco de las Tecnologías de la Información y la Comunicación están surgiendo nuevas formas de planificar y desarrollar los procesos de Enseñanza-Aprendizaje, de manera que se promueva la participación sincrónica y asincrónica entre los sujetos que no se encuentran físicamente en el mismo lugar, ni en las mismas coordenadas temporales (pág. 1).

La guía que desarrolla el docente resulta fundamental, al momento de transmitir conocimientos, es de vital importancia utilizar los recursos adecuados, materiales didácticos y estrategias innovadoras para atender a la diversidad de estudiantes permitiendo a los mismos desarrollar sus destrezas de una manera autónoma que puedan hacer frente a los retos del cotidiano 
Instituto Internacional de Investigación y Desarrollo Tecnológico Educativo INDTEC, C.A.

DOI: https://doi.org/10.29394/Scientific.issn.2542-2987.2020.5.17.19.350-369

OAI-PMH: http://www.indteca.com/ojs/index.php/Revista_Scientific/oai

Ensayo Original / Original Essay

vivir.

Acorde con esto, Hernández (2017) añade en la implementación de las

TIC a la educación que:

Dentro de los roles que asumen cada agente educativo, los estudiantes actuales, utilizan las herramientas tecnológicas para facilitar el aprendizaje; esta evolución surgió desde las primeras concepciones con la calculadora, el televisor, la grabadora, entre otras; sin embargo, el progreso ha sido tal que los recursos tecnológicos se han convertido en recursos educativos, donde la búsqueda por mejorar el aprendizaje trae consigo la tarea de involucrar la tecnología con la educación. Y es con la docencia que se viene completando el proceso de enseñanza-aprendizaje [...] (pág. 329).

Por lo tanto, se deben aprovechar estos recursos que se tiene hoy en día, pues de esta manera se genera la accesibilidad a todas las personas, fomentando la innovación y avance con éxito en el sistema educativo. El manejo de las tecnologías de la información y comunicación en la pedagogía se centra en el estudiante, por otro lado, el docente sirve de orientador de los discentes, brindándoles una retroalimentación oportuna que garantice el avance. Además, los estudiantes no solo receptan conocimientos, pues aportan a construir aprendizajes significativos.

Es importante considerar el rol del docente al momento de implementar las TIC ya que no se trata de brindarles el conocimiento "exacto" a sus estudiantes, sino proporcionarles la guía para que seleccionen y accedan a una información fiable, así como brindarles las pautas para que accedan al conocimiento y puedan citar correctamente, respetando el derecho de autor y no caer en el plagio, por lo tanto, la práctica de los valores es más fuerte en el uso de la virtualidad. Mencionando a Martínez (2016b):

El docente debe asumir su nuevo rol dentro del proceso educativo, a fin de brindar una educación de calidad. La formación inicial y permanente del profesorado en materia de tecnología es fundamental para garantizar su adecuación al 
Instituto Internacional de Investigación y Desarrollo Tecnológico Educativo INDTEC, C.A. DOI: https://doi.org/10.29394/Scientific.issn.2542-2987.2020.5.17.19.350-369

OAI-PMH: http://www.indteca.com/ojs/index.php/Revista_Scientific/oai

Ensayo Original / Original Essay

entorno educativo en el cual deberá desarrollar su tarea docente. En tal sentido, la aplicación de las TIC en la educación, exige que el docente participe en programas de formación permanente, para que de esta manera, logre un buen desenvolvimiento en cuanto al uso en los procesos de enseñanza-aprendizaje y alcance las competencias, habilidades y conocimientos necesarios que le permitan incorporar y manejar eficientemente con creatividad $y$ autonomía la tecnología como un recurso más en las áreas de desarrollo del currículo (pág. 102).

Por esta razón resulta fundamental conocer el paradigma actual para poder orientarnos de la manera correcta y ya no segregar al estudiante o realizar prácticas educativas erróneas que minimicen al estudiante al momento de compararlo y querer que sea igual a los demás, solo a través del conocimiento del nuevo paradigma y el uso adecuado de las tecnologías estaremos preparados y nos dará las pautas para actuar de la manera correcta y así valorar la diversidad de los estudiantes que podemos encontrar en los distintos contextos educativos, manteniendo siempre presente la ética profesional.

La labor del docente no es solo impartir conocimientos, también radica una gran responsabilidad y compromiso de velar por la estabilidad emocional de cada estudiante y esto solo se logrará a través de potenciar el verdadero valor de las capacidades y habilidades que poseen cada uno, permitiéndoles trabajar colaborativamente en equipo y sacar a flote las mismas, donde sientan que son parte del equipo y no solo un integrante más, por medio de esta interacción comprenderán la importancia del apoyo de los unos a hacia los otros para poder obtener resultados positivos y que todos sean actores activos para lograr grandes éxitos en su vida diaria y cambiar la mirada de la sociedad actual donde solo se ha juzgado y criticado por las limitaciones que posee una persona y no se ha valorado lo más importante que es el ser en sí, sus sentimientos, sus anhelos, sus sueños y las capacidades que puede ofrecer a 
esta sociedad.

El aprendizaje debe ser profundo, en el que la misión del docente sea desarrollar ambientes y contextos flexibles; diseñando las actividades en función de la variabilidad existente en el aula. Es decir, los docentes deben reflexionar sobre sus prácticas educativas, teniendo en cuenta que no es mejor por el hecho de dar gran cantidad de contenidos, sino al verificar si estos aprendizajes son significativos a lo largo de la vida de los estudiantes, brindándoles un aprendizaje oportuno que permita enfrentarse posteriormente de mejor manera a los niveles de exigencia de cada curso a seguir en el plano educativo y la sociedad en que se encuentren inmersos.

La responsabilidad que existe entre los docentes y los estudiantes debe ser compartida, es decir fomentar en el alumno ese empoderamiento, brindándole protagonismo al mismo, cambiando los roles, dando el poder en el margen del respeto.

Es importante ofrecer a los estudiantes entornos acogedores y seguros, que les permitan desenvolverse con autonomía y confianza, al mismo tiempo de facilitar el desarrollo de los aprendizajes, pues deben ser tratados de la manera correcta, sintiéndose incluidos en sus clases, participando con todos sus compañeros, aumentado su autoestima, responsabilidad, seguridad personal, mejorando así su conducta y comportamiento.

Lograr un sistema educativo equitativo y de calidad no es sencillo, y exige un arduo trabajo, que tiene implicaciones políticas, económicas y curriculares en la aceptación del alumnado, en la formación continua del profesor para que este tenga capacidad de innovación, en la aplicación de las tecnologías, e incluso en la instrucción e inclusión de las familias, cuyo rol es cada vez más crucial en la adquisición de las diferentes destrezas académicas y sociales. 


\section{Conclusiones}

En síntesis, para que se pueda brindar en las instituciones educativas una educación que cumpla con las expectativas de los estudiantes atendiendo a la época actual en la que se están desarrollado, es necesario contar con los factores de acceso como lo son, la implementación de equipos tecnológicos en los establecimientos y en los hogares en la actualidad. Es necesario realizar cambios de manera oportuna en los diferentes métodos de enseñanzaaprendizaje, que los docentes se capaciten para ofrecer una mejor preparación a los educandos insertos en el sistema educativo, empleando los instrumentos adecuados para la introducción correcta de estas tecnologías, mediante ellas tenemos el acceso a diferentes recursos, plataformas, información, comunicación entre otros beneficios y así poder motivar a los estudiantes atendiendo sus necesidades de acuerdo a sus intereses.

\section{Referencias}

Arza, J. (2010). Familia y nuevas tecnologías: Cómo ayudar a los menores para que hagan un buen uso de la televisión, el teléfono móvil, los videojuegos e Internet. España: Consejo Audiovisual de Navarra CoAN. Recuperado de:

https://www.educacion.navarra.es/documents/27590/585666/Familia NuevasTecnologias.pdf

Becerra, C. (2019). EI uso de TIC, TAC y TEP: Una asignatura aún pendiente para el profesorado de inglés. Trabajo de grado. España: Universidad de La Laguna. Recuperado de: https://riull.ull.es/xmlui/handle/915/16809

Belloch, C. (2012a,b). Las Tecnologías de la Información y Comunicación en el aprendizaje. Material docente [on-line]. España: Departamento de Métodos de Investigación y Diagnóstico en Educación de la Universidad de Valencia. Recuperado de: 


\section{http://www.uv.es/bellochc/pedagogia/EVA1.pdf}

Brito, V. (2004). El foro electrónico: una herramienta tecnológica para facilitar el aprendizaje colaborativo. Edutec: Revista Electrónica de Tecnología Educativa, 17, 1-12, e-ISSN: 1135-9250. Recuperado de: https://doi.org/10.21556/edutec.2004.17.532

Cabero, J., \& Fernández, J. (2014). Una mirada sobre las TIC y la Educación Inclusiva. Comunicación y Pedagogía: nuevas tecnologías y recursos didácticos, (279-280), 38-42, ISSN: 1136-7733. España: Centro de Comunicación y Pedagogía.

Forero, I. (2009). La Sociedad del Conocimiento. Revista Científica General José María Córdova, 5(7), 40-44, e-ISSN: 1900-6586. Recuperado de: https://www.redalyc.org/articulo.oa?id=476248849007

García, F., Gértrudix, F., Durán, J., Gamonal, R., \& Gálvez, M. (2011). Señas de identidad del "nativo digital". Una aproximación teórica para conocer las claves de su unicidad. Cuadernos de documentación multimedia, 22, 110-127, e-ISSN: 1575-9733. Recuperado de: https://doi.org/10.5209/rev CDMU.2011.v22.38339

Hernández, R. (2017). Impacto de las TIC en la educación: Retos y Perspectivas. Propósitos y Representaciones, 5(1), 325-347, e-ISSN: 2310-4635. Recuperado de:

http://dx.doi.org/10.20511/pyr2017.v5n1.149

Martínez, O. (2018). Uso de las Tecnologías de la Información y la Comunicación en la Educación Básica. Revista Scientific, 3(10), 154174, e-ISSN: 2542-2987. Recuperado de:

https://doi.org/10.29394/Scientific.issn.2542-2987.2018.3.10.8.154-174 Martínez, O. (2016a,b). Programa de Formación Docente de las Tecnologías del Aprendizaje y el Conocimiento (Tac) en la Universidad Pedagógica Experimental Libertador Núcleo Barinas (Venezuela). Revista Scientific, 1(1), 90-114, e-ISSN: 2542-2987. 
Recuperado de: https://doi.org/10.29394/scientific.issn.25422987.2016.1.1.6.90-114

Martín, A., \& Montilla, M. (2016). El uso del blog como herramienta de innovación y mejora de la docencia universitaria Profesorado. Revista de Currículum y Formación de Profesorado, 20(3), 659-686, eISSN: 1138-414X. Recuperado de:

https://www.redalyc. org/articulo.oa?id=56749100015

Mora, F. (2012). Posibilidades educativas de la wiki. Revista Tecnología en Marcha, 25(3), 113-118, e-ISSN: 2215-3241. Recuperado de: https://revistas.tec.ac.cr/index.php/tec marcha/article/view/463

Roncancio-Ortiz, A., Ortiz-Carrera, M., Llano-Ruiz, H., Malpica-López, M., \& Bocanegra-García, J. (2017). El uso de los videojuegos como herramienta didáctica para mejorar la enseñanza-aprendizaje: una revisión del estado del tema. Revista Ingeniería, Investigación y Desarrollo, 17(2), 36-46, e-ISSN: 2422-4324. Recuperado de: https://doi.org/10.19053/1900771X.v17.n2.2017.7184

Zambrano, F., \& Balladares, A. (2017a,b). Sociedad del conocimiento y las TEPs. INNOVA Research Journal, 2(10), 169-177, e-ISSN: 2477-9024. Recuperado de: https://doi.org/10.33890/innova.v2.n10.2017.534 
Ensayo Original / Original Essay

\section{Silvia Yesenia Andrade Parra}

e-mail: silvi yesse01@outlook.es

Nacida en Cuenca, Ecuador, el 18 de diciembre del año

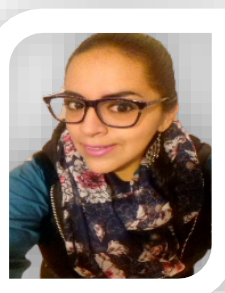
1993. Licenciada en Ciencias de la Educación, Mención Psicología Educativa por la Universidad Católica de Cuenca (UCACUE); Docente de la Unidad Educativa "Las Palmas". 
Ensayo Original / Original Essay

Martha Jhamiled Tapia Tapia

e-mail: jhamytapia@hotmail.com

Nacida en Cuenca, Ecuador, el 14 de diciembre del año

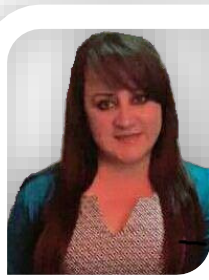

1979. Licenciada en Ciencias de la Educación, Mención

Inglés por la Universidad Técnica Particular de Loja (UTPL); Docente de la Unidad Educativa "Las Palmas". 
DOI: https://doi.org/10.29394/Scientific.issn.2542-2987.2020.5.17.19.350-369

OAI-PMH: http://www.indteca.com/ojs/index.php/Revista_Scientific/oai

Ensayo Original / Original Essay

\section{Flor del Cisne Tituana Vásquez}

e-mail: tituanaflor@gmail.com

Nacida en Zaruma, Ecuador, el 6 de enero del año 1986.

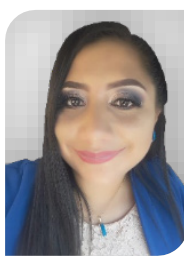

Licenciada en Ciencias de la Educación, Mención en Educación Básica por la Universidad Técnica Particular de Loja (UTPL); Docente de la Unidad Educativa "Dr. Ernesto

\section{A. Castro".}

